\title{
Lose appetite, lose control: integrins and noncanonical autophagy regulate germinal center reactions
}

\begin{abstract}
Jeremy S. Leventhal Bronx, New York, USA.
\end{abstract}

Division of Nephrology, Department of Medicine, and Transplant Research Institute, Icahn School of Medicine at Mount Sinai, New York, New York, USA. Renal Division, James J. Peters VA Medical Center, imentally can be complex, given the reliance on LC3 for most readouts and the overlap of this marker with both forms of autophagy. Molecular analysis of noncanonical autophagy complexes identified the essential protein RUN and cysteine-rich domain containing beclin 1 interacting protein (Rubicon). Rubicon is involved only in noncanonical autophagy and has the potential to inhibit canonical autophagy (6). Although the first observations of noncanonical autophagy were in the setting of pathogen clearance and effector immune responses, subsequent observations noted that noncanonical autophagy also plays a role in maintaining humoral tolerance. Mice with myeloid cells lacking canonical autophagy proteins that overlap with LAP and mice lacking Rubicon, which are unable to process phagocytosed apoptotic cells, were shown to develop a lupus-like syndrome. Conversely, mice lacking proteins, such as Unc-51-like kinase 1 (ULK1), only found in the canonical autophagy pathway did not develop lupus-like disease (7).

\section{Canonical and noncanonical} autophagy and $B$ cell function

The story of autophagy in B cells is complex and depends on the subset of B cells studied. Our lab built on the seminal findings of Chen et al. and demonstrated that allospecific memory B cells (Bmem) are dependent on autophagy $(8,9)$. Neither group differentiated between involvement of canonical and noncanonical autophagy; however, the abnormalities in oxidative stress and mitochondrial fitness in ATG7-null Bmem point to a deficiency of canonical autophagy (8). Conversely, during germinal center (GC) formation, canonical autophagy is relatively suppressed and noncanonical autophagy is upregulated in B cells (10). Using chloroquine and bafilomycin A1 to measure the proportion of autophagy that is canonical versus noncanonical, Martinez-Martin et al. demonstrated that deletion or mutation of canonical autophagy protein WD repeat 
domain, phosphoinositide interacting 2 (WIPI2), had two measurable effects: increased noncanonical autophagy and decreased antigen-specific GC B cell formation (10).

The question remained, is there a relationship between noncanonical autophagy and GC responses? Interestingly, Martinez-Martin and colleagues generated GC responses using viral particles or TLR9 ligand CpG molecules as a stimulus; therefore, it could be hypothesized that WIPI2 deficiency impairs GC responses due to a concurrent increase in noncanonical autophagy. If this were true, impairing noncanonical autophagy in B cells would impair GC responses. In this issue, Raso et al. definitively answer this question by demonstrating accentuated GC responses in mice reconstituted with Rubicondeleted B cells (11). Moreover, these data are supported further by observations of a preferential increase of noncanonical autophagy in GC B cells compared with follicular B cells, which did not increase noncanonical autophagy in response to TLR9 or TLR4 stimulation. This finding builds on previous work and more clearly defines how TLR ligands and noncanonical autophagy work to shape B cell responses.

\section{TLR ligands, integrins, and GC reactions}

It has been established that $\mathrm{B}$ cell responses are amplified by concurrent innate immune receptor and $\mathrm{B}$ cell receptor (BCR) signaling, which link innate and adaptive immune systems (12). In 2016, work from Acharya et al. established that TLR-induced increases in antibody production and proliferation by marginal zone (MZ) and B-1 B cells are regulated by integrin $\alpha_{v} \beta_{3}$ (13). Integrins are membrane-bound proteins that can transmit signals from extracellular to intracellular compartments through interactions with the cytoskeleton and signal transduction molecules (14). In MZ B cells, loss of the integrin $\alpha_{v} \beta_{3}$ prolongs TLR downstream signaling through aborted LC3 and autophagyrelated 5-dependent (ATG5-dependent) degradation of TLR-containing endosomes (13). This produces a robust but unregulated $\mathrm{B}$ cell response that is accompanied by generation of autoantibodies (13).

Raso et al. determined that a similar phenomenon also occurs in $\mathrm{T}$ celldependent GC responses. In this case, not only are antibody levels higher (specifi- cally IgG2c isotype), but GC cells undergo increased affinity maturation, as evidenced by a significantly higher number of mutations in sequenced IgG heavy chains (11). Consistent with this, genes involved in somatic hypermutation, IgG2c class switch, and plasma cell differentiation were more highly expressed in $\alpha_{\mathrm{v}} \beta_{3}$-deficient, antigenspecific GC B cells (11). Impressively, the associated increase in mutations produced antibodies with higher affinity and greater breadth of antigen diversity, and mice with $\alpha_{\mathrm{v}} \beta_{3}$-deficient $\mathrm{B}$ cells produced higher titers of anti-HA to both PR/8 and Cal-09 antigens compared with control mice (11). The exaggerated GC response not only increased antibody titers, but also generation of antigenspecific long-lived memory cells. Raso et al. also demonstrated enhanced memory recall responses that are independent of TLR adjuvants in response to secondary challenge, suggesting an intrinsic difference in GC reactions of $\alpha_{v} \beta_{3}$-null $B$ cells that affect shortand long-lived humoral responses.

\section{Conclusions}

The work of Raso et al. has tremendous implications for clinical medicine, while also raising additional mechanistic questions. For years, models of autoimmune disease in mice have been generated by injecting a bacterial extract, CFA, along with the selfpeptide target, to generate disease phenotypes. Could autoimmunity in humans arise from inherited or acquired problems with the $\alpha_{v} \beta_{3}$ integrin in the setting of bacterial infection? More to the point, could we limit unwanted $\mathrm{B}$ cell responses by affecting the $\alpha_{v} \beta_{3}$ integrin or, farther downstream, noncanonical autophagy? In their work, Raso et al. demonstrate the converse potential of their delineated mechanism; naive mice lacking $\alpha_{v} \beta_{3}$ in B cells survived, whereas control mice died, after influenza infection. Inhibiting $\alpha_{v} \beta_{3}$ or noncanonical autophagy in patients with sepsis may provide a life-preserving boost to the adaptive immune system. These possibilities are attractive and exciting, but require further understanding of the integrin-B cell phenomenon. For example, how does increased NF- $\kappa$ B activity lead to increased B cell responses? The effect may be intrinsic to the B cell or involve complex interactions with other immune cells. One possibility is that increased NF- $\mathrm{NB}$ activation mediates IL-6 production that in turn encourages differentiation of $\mathrm{T}$ follicular helper cells, as observed in B cells from mice lacking liver kinase B1 (LKB1) (15). Regardless, the findings by Raso et al. are a major leap forward in our understanding of how GC responses, integrins, innate immune receptors, and noncanonical autophagy interact to shape the immune responses at the heart of human health.

Address correspondence to: Jeremy S. Leventhal, Icahn School of Medicine at Mount Sinai, 1 Gustave L. Levy Place, Box 1243, New York, New York 10029, USA. Phone: 212.241.8539; Email: Jeremy.leventhal@mssm.edu.

1. Choi AM, Ryter SW, Levine B. Autophagy in human health and disease. N Engl JMed. 2013;368(7):651-662.

2. Klionsky DJ, et al. Guidelines for the use and interpretation of assays for monitoring autophagy (3rd edition). Autophagy. 2016;12(1):1-222.

3. Codogno P, Mehrpour M, Proikas-Cezanne T. Canonical and non-canonical autophagy: variations on a common theme of self-eating? Nat Rev Mol Cell Biol. 2011;13(1):7-12.

4. Sanjuan MA, et al. Toll-like receptor signalling in macrophages links the autophagy pathway to phagocytosis. Nature. 2007;450(7173):1253-1257.

5. Takeshige K, Baba M, Tsuboi S, Noda T, Ohsumi Y. Autophagy in yeast demonstrated with proteinase-deficient mutants and conditions for its induction. J Cell Biol. 1992;119(2):301-311.

6. Zhong Y, et al. Distinct regulation of autophagic activity by Atg14L and Rubicon associated with Beclin 1-phosphatidylinositol-3-kinase complex. Nat Cell Biol. 2009;11(4):468-476.

7. Martinez J, et al. Noncanonical autophagy inhibits the autoinflammatory, lupus-like response to dying cells. Nature. 2016;533(7601):115-119.

8. Chen M, et al. Essential role for autophagy in the maintenance of immunological memory against influenza infection. Nat Med.2014;20(5):503-510.

9. Fribourg M, Ni J, Nina Papavasiliou F, Yue Z, Heeger PS, Leventhal JS. Allospecific memory B cell responses are dependent on autophagy. $A m J$ Transplant. 2018;18(1):102-112.

10. Martinez-Martin N, et al. A switch from canonical to noncanonical autophagy shapes B cell responses. Science. 2017;355(6325):641-647.

11. Raso $F$, et al. $\alpha_{v}$ Integrins regulate germinal center $B$ cell responses through noncanonical autophagy. JClin Invest. 2018;128(9):4163-4178.

12. Leadbetter EA, Rifkin IR, Hohlbaum AM, Beaudette BC, Shlomchik MJ, Marshak-Rothstein A. Chromatin-IgG complexes activate $B$ cells by dual engagement of IgM and Toll-like receptors. Nature. 2002;416(6881):603-607.

13. Acharya M, et al. av Integrins combine with LC3 and atg 5 to regulate Toll-like receptor signalling in B cells. Nat Commun. 2016;7:10917.

14. Hynes RO. Integrins: bidirectional, allosteric signaling machines. Cell. 2002;110(6):673-687.

15. Walsh NC, et al. LKB1 inhibition of NF- $\mathrm{kB}$ in B cells prevents $\mathrm{T}$ follicular helper cell differentiation and germinal center formation. EMBO Rep. 2015;16(6):753-768. 